\title{
Rezension: Clemens Schwender, Werner F. Schulz und Martin Kreeb (Hrsg.): Medialisierung der Nachhaltigkeit. Das Forschungsprojekt balance[f]: Emotionen und Ecotainment in den Massenmedien, Metropolis Verlag, Marburg 2008, 491 Seiten.
}

\author{
Peter Schmuck
}

Online veröffentlicht: 26.2.2009

(C) Springer-Verlag 2009

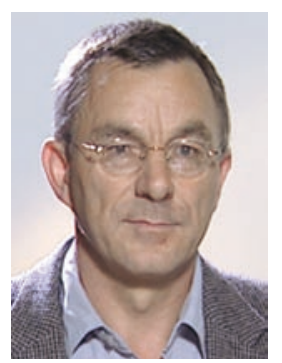

Der Sammelband behandelt ein existentielles Thema unserer Zeit: Wie können die Produktions- und Konsumtionsmuster und insgesamt die Lebensweise von Menschen im 21. Jahrhundert derart umgestaltet werden, dass künftige Generationen ähnlich gute Lebens-Chancen haben wie die heute lebenden Menschen? Welche Rolle können Massenmedien in dieser Transformation spielen? Diese Thematik wird seit etwa 20 Jahren von verschiedenen Interessengruppen unserer Gesellschaft hier in Deutschland und international unter dem terminologischen Flaggschiff der „Nachhaltigkeit“ diskutiert. Bei der existentiellen Bedeutsamkeit des Themas verwundert es nicht, dass zwar weithin Konsens über die Vision einer gerechten und ökologisch balancierten Gesellschaft besteht, jedoch Dissens über gangbare und sinnvolle Wege zu verzeichnen ist, welche zu Nachhaltiger Entwicklung tatsächlich substantiell beizutragen vermögen.

Vor diesem Hintergrund besteht ein Verdienst des Buches zunächst darin, in einem ersten Wurf die äußerst komplexen Zusammenhänge zwischen Zielvorgaben von Staat, Ver-

Prof. Dr. Peter Schmuck $(\bowtie)$

Leiter des Instituts für Nachhaltigkeit und Umweltpolitik

University of Management and Communication UMC Potsdam (FH)

Am Kanal 16-18, 14467 Potsdam, Deutschland

E-Mail: p.schmuck@umc-potsdam.de bänden, NGOs und Unternehmen und den Rezipienten entsprechender Informationen, vermittelt über die Medien des Internets, der Presse und des Fernsehens zu skizzieren und Forschungsaufgaben für Marketing-, Medien- und Konsumwissenschaft herauszuarbeiten. Ein weiteres Verdienst der Autoren des Buches darf man darin sehen, dass aus dieser Skizze ein sehr facettenreiches Aktions- und Forschungsprogramm konzipiert wurde, in dem von unterschiedlichen Seiten aus neue Wege der Nachhaltigkeitskommunikation ersonnen und zum Teil umgesetzt und wissenschaftlich bewertet werden. Es werden aufregende Fragen gestellt - wie beispielsweise „Sind Nachhaltigkeitsthemen im Fernsehen ein Quotenkiller?" - und empirisch begründete Argumente für die nicht erwartete Antwort - „Nein!“ - gesammelt. Es werden innovative Vorschläge gemacht, z. B. die Nachhaltigkeitsthematik mit positiven Emotionen assoziiert in Medien und Werbung zu bringen - und diese Vorschläge werden in verschiedenen Praxisprojekten wie der Gestaltung entsprechender Fernsehsendungen oder einer Jugendzukunftskonferenz umgesetzt.

Schließlich wird über diese vielen Aktivitäten, die im Rahmen des Forschungsprojektes Balance[f] und den davon bereits inspirierten Arbeiten außerhalb des Projektes zwischen 2002 und 2008 entstanden sind, gut strukturiert berichtet. Dass dabei unterschiedliche Stil-Formate bei den einzelnen Kapiteln (vom Essay bis zum Fachartikel) vorzufinden sind, spiegelt die unterschiedlichen Akteursgruppen wider, welche an einem solchen komplexen Unterfangen beteiligt sind.

Insgesamt ist das Buch eine Fundgrube für Nachhaltigkeitsakteure in Wissenschaft, Politik, Organisationen und Unternehmen, welche sich bei der Kommunikation von Nachhaltigkeitsthemen bislang weitgehend auf ihre Intuitionen verlassen mußten. Mit dem Band haben die Autoren ein erstes zusammenhängendes Werk mit wissenschaftlichem Anspruch zu Chancen und Grenzen der Medialisierung der Nachhaltigkeit vorgelegt. 